\title{
Talk2Me: Uma abordagem computacional para auxiliar na identificação de falhas no processo de alfabetização
}

\author{
Warley Almeida Silva ${ }^{1}$, Jorão Gomes Jr. ${ }^{2}$, Igor de O. Knop ${ }^{1}$, \\ Eduardo Barrére ${ }^{2}$, Jairo Francisco de Souza ${ }^{2}$ \\ ${ }^{1}$ Departamento de Ciência da Computação - Universidade Federal de Juiz de Fora (UFJF) \\ 36.360-900 - Juiz de Fora - MG - Brasil \\ ${ }^{2}$ Programa de Pós-Graduação em Ciência da Computação - (UFJF) \\ 36.360-900 - Juiz de Fora - MG - Brasil \\ \{joraojunior, warley.almeida,igorknop\}@ice.ufjf.br \\ \{eduardo.barrere, jairo.souza\}@ice.ufjf.br
}

\begin{abstract}
Being able to read fluently directly affects the individual's interaction with society. However, there are few tools that help in the process of developing fluency and in the diagnosis of problems in a playful way. Although applied to foreign language learning, automatic speech recognition (ASR) techniques are not widely used in the literature to support mother tongue development. In this sense, this work proposes a gamified computational approach to diagnose failures in literacy. The proof of concept shows that the tool developed is capable of automatically producing reports that are consistent with reality and useful for teachers and managers in decision-making.
\end{abstract}

Resumo. Ser capaz de ler com fluência afeta diretamente a interação do indivíduo com a sociedade. No entanto, existem poucas ferramentas que ajudam no processo de desenvolvimento da fluência e no diagnóstico de problemas de forma lúdica. Embora sejam aplicadas em aprendizado de língua estrangeira, técnicas de reconhecimento automático de fala (ASR) não são muito utilizadas na literatura para auxiliar o desenvolvimento da língua materna. Neste sentido, este trabalho propõe uma abordagem computacional gamificada para diagnosticar falhas na alfabetização. A prova de conceito mostra que a abordagem desenvolvida é capaz de produzir automaticamente relatórios condizentes com a realidade e úteis para professores e gestores na tomada de decisões.

\section{Introdução}

Ser capaz de ler com fluência não é apenas um requisito estabelecido pela Base Nacional Comum Curricular (BNCC) [MEC 2018], mas também um fator de alta importância para a inserção e participação ativa do indivíduo na sociedade [Dias et al. 2016]. A importância da fluência em leitura na vida dos indivíduos desperta um aumento na produção científica que discute métodos de avaliação da leitura, de acordo com o levantamento realizado por [Suehiro et al. 2009]. Uma das principais razões apontadas para a dificuldade de avaliação de leitura é a falta de instrumentos que ajudem no processo [Suehiro et al. 2009]. Esforços recentes têm proposto ferramentas que atuem na avaliação da fluência em larga escala através de técnicas de reconhecimento automático 
VIII Congresso Brasileiro de Informática na Educação (CBIE 2019)

Anais do XXX Simpósio Brasileiro de Informática na Educação (SBIE 2019)

de fala (ASR) [Soares et al. 2018], mas faltam ferramentas que auxiliem os professores no diagnóstico mais local do desenvolvimento da fluência dos alunos na leitura, uma vez que não é humanamente possível que um professor mantenha um acompanhamento de todos os alunos a fim de atenuar ou abolir todos os seus problemas [Celestino 2019].

Nesse contexto, o lúdico aparece com uma alternativa para auxiliar os professores no acompanhamento do desenvolvimento dos alunos na fluência em leitura, uma vez que se mostra como uma ferramenta essencial para que as crianças possam desenvolver seu aprendizado [Rocha 2018]. O lúdico pode ser entendido como o uso de jogos, brinquedos ou brincadeiras na consolidação de conhecimentos, de forma que a criança possa aprender conceitos através das atividades desempenhadas [Dallabona and Mendes 2004]. Em conjunto com técnicas de gamificação, que têm sido aplicadas com sucesso em diversas áreas da educação [Santos and Freitas 2017] e consistem na utilização de características presentes em jogos para motivar os participantes, a utilização de jogos em atividades de diagnóstico da fluência dos alunos em leitura tem potencial para estimular um maior engajamento dos alunos e tornar as atividades menos cansativas.

Nessa visão, este trabalho tem como objetivo apresentar uma abordagem computacional que auxilie professores no diagnóstico da capacidade de decodificação das palavras nos anos iniciais do ensino fundamental. Esta abordagem faz uso de um jogo para a coleta lúdica do material, sistemas de ASR para a avaliação da fluência e técnicas de gamificação para engajar os alunos durante a atividade. Com base nas informações colhidas pela implementação da abordagem, relatórios sobre desempenho dos alunos são disponibilizados para uso de professores e gestores na tomada de decisões.

Este artigo segue assim organizado: na Seção 2 são apresentados a fundamentação teórica e os trabalhos relacionados; na Seção 3 é apresentada a abordagem computacional proposta neste trabalho; na Seção 4 são apresentados o jogo e a arquitetura desenvolvida; na Seção 5 é apresentada uma prova de conceito da abordagem proposta; e na Seção 6 são apresentados considerações finais e futuros trabalhos relacionados ao tema.

\section{Uso de gamificação para avaliação de fluência}

De acordo com [Fuchs et al. 2001], a fluência em leitura pode ser definida como a habilidade com que um individuo traduz oralmente um texto com velocidade e precisão através da rapidez no reconhecimento das palavras e a segmentação correta dos fonemas pronunciados. Nesse sentido, três elementos principais são necessários para considerar uma pessoa fluente: precisão na leitura, boa taxa de leitura de palavras e prosódia durante a pronúncia [Hudson et al. 2005]. Durante a alfabetização, as crianças obtêm fluência de acordo com esses três elementos e são ensinados pela figura docente. No entanto, é humanamente impossível suprir as dificuldades de todos os alunos, deixando muitas vezes o desenvolvimento da linguagem oral defasada [Celestino 2019].

Visto que a fluência em leitura está estritamente ligada com a habilidade de se pronunciar, caso não existam iniciativas capazes de lidar com a grande quantidade de alunos e validar a forma com que está ocorrendo o ensino da fala, a capacidade de se comunicar corretamente fica comprometida desde a infância. A falta de ferramentas que auxiliem o processo de avaliação da fluência em leitura é uma das principais dificuldades apontadas por [Suehiro et al. 2009]. Portanto, torna-se necessário produzir ferramentas que atendam as necessidades dos professores no que diz respeito a avaliação de fluência. 
VIII Congresso Brasileiro de Informática na Educação (CBIE 2019)

Anais do XXX Simpósio Brasileiro de Informática na Educação (SBIE 2019)

A utilização de conceitos de gamificação tem potencial para aumentar os resultados alcançados com ferramentas de avaliação de fluência. Nos últimos anos, a gamificação tem sido amplamente utilizada no âmbito da educação para engajar os alunos em atividades diversas [Santos and Freitas 2017]. No contexto de educação, gamificação pode ser definida como a aplicação de elementos de jogos digitais com o intuito de tornar o processo de aprendizagem mais atrativo [Barrére et al. 2017]. Técnicas de gamificação têm alcançado bons resultados tanto no mercado quanto na academia, de forma que sua aplicação tenha crescido exponencialmente nos últimos anos e a consolidação de uma base teórica do que é gamificação tem sido o foco da comunidade acadêmica [Deterding et al. 2011].

No que diz respeito a avaliação automática de fluência, percebe-se que existe uma tendência em aplicar gamificação na avaliação da fluência em leitura com foco no aprendizado de uma segunda língua. Nesse sentido, [Tejedor-García et al. 2016] propõem uma ferramenta chamada TipTop Talk!, que auxilia no desenvolvimento da pronúncia de uma segunda língua. A gamificação é aplicada através de pontos, troféus e outras conquistas com o intuito de motivar o estudo continuado do idioma. No entanto, as técnicas de ASR utilizadas são externas ao projeto e no modelo caixa-preta. Ainda, não são realizadas avaliações do impacto da ferramenta numa situação real.

Quando técnicas de gamificação são aplicadas na avaliação da fluência em leitura da língua materna, existe um grande foco no auxílio de crianças com dificuldades oriundas de transtornos ou deficiências físicas. Nesse sentido, [Anwar et al. 2011], [Liu et al. 2015] e [Saputra 2015] propõem ferramentas para auxiliar no desenvolvimento da fluência em leitura em crianças autistas, com dificuldades auditivas e disléxicas, respectivamente. As técnicas de gamificação são utilizadas para manter a atenção durante as atividades e motivá-las, de forma a superar as dificuldades oriundas da sua condição. Todos os trabalhos apresentam avaliações das ferramentas através de questionários para as crianças envolvidas, mas a tecnologia de ASR utilizada não é mencionada ou não é autoral, o que limita a possibilidade de extração de informações relativas quanto a forma de leitura das crianças.

Por outro lado, [Takita et al. 2017] e [Musti-Rao et al. 2015] discutem ferramentas que auxiliam na avaliação da fluência em leitura na língua materna para todos os alunos, em especial aqueles que apresentam dificuldades de leitura por questões de desenvolvimento. Na ferramenta proposta por [Takita et al. 2017], palavras são apresentadas na tela e a criança deve pronunciá-las, recebendo um resultado instantâneo se a pronúncia foi fluente, não fluente ou meio termo. [Musti-Rao et al. 2015] faz uso de um aplicativo disponível na App Store. Os dois trabalhos apresentam uma avaliação quantitativa e qualitativa das ferramentas utilizadas, embora o domínio sobre o código fonte da ferramenta, tanto do aplicativo quanto das técnicas de ASR, seja limitado.

Após uma análise das ferramentas propostas, constata-se que as soluções raramente apresentam resultados da sua aplicação com dados reais e que nenhuma adaptação pode ser realizada nos módulos de ASR dessas soluções, o que impacta diretamente no retorno que a atividade pode trazer para a atividade docente. Existem também poucos esforços que procuram diagnosticar dificuldades de leitura em alunos que não possuem nenhum tipo de deficiência ou dificuldade. Além disso, as informações colhidas através das ferramentas analisadas não são fornecidas para responsáveis que podem modificar a 
situação atual através de relatórios ou análises. Nesse sentido, este trabalho contribui para a área por apresentar uma abordagem que fornece informações ricas através do uso de um módulo de ASR especializado na avaliação de leitura e auxiliar professores no diagnóstico de crianças com dificuldades de leitura que poderiam passar despercebidas.

\section{Talk2Me: abordagem para avaliação de fluência}

Nesta seção é apresentada a abordagem para avaliação de fluência proposta neste trabalho. O objetivo principal da abordagem é fornecer formas de diagnóstico para professores, diretores e responsáveis na identificação prévia de dificuldades enfrentadas pelos alunos, de forma que as mesmas sejam tratadas posteriormente. A abordagem Talk2Me é composta por três etapas principais:

Coleta de áudios: Embora seja uma tarefa essencial para o funcionamento da abordagem, a coleta de áudios não é trivial. Motivar a participação do aluno na atividade, mesmo que esta não seja avaliativa, requer da aplicação uma interface de coleta que seja interessante. Por esse motivo, optou-se por desenvolver um jogo, chamado SpaceGEMS, que faz uso de conceitos de gamificação para realizar a coleta dos áudios de maneira implícita. Para que alguma ação seja realizada dentro do jogo, o aluno deve selecionar uma palavra associada a uma ação e gravar a leitura. Portanto, a atenção do aluno é deslocada do processo de captura de áudio e direcionada para a dinâmica do jogo aplicada pelo professor. Em conjunto com a aplicação do lúdico, pretende-se aumentar o engajamento dos alunos na participação e diminuir a tensão associada com um processo avaliativo.

Processamento de áudios: Os áudios coletados durante a atividade são posteriormente avaliados com o auxílio de um sistema de ASR. Cada áudio é separadamente transcrito e, com base num conjunto pré-determinado de pronúncias aceitáveis e não aceitáveis, cada palavra é determinada como lida correta ou incorretamente.

Geração de resultados: Com base nos resultados adquiridos pela avaliação dos áudios, resultados micros e macros podem ser obtidos. A partir de um esquema de pontuação, valorizando acertos e penalizando erros, é possível construir um ranking de pontuação para os alunos de uma turma e o conjunto de palavras mais erradas e acertadas dentro de uma turma. Já para um determinado aluno, destaca-se um ou mais fonemas que são frequentemente pronunciados incorretamente ou dificuldades em marcar a sílaba tônica.

O funcionamento do jogo e dinâmica de coleta de áudios, bem como a arquitetura de software que conecta o jogo com os sistemas de ASR e geração de resultado, são explicados na Seção 4. A partir dos resultados levantados ao final da abordagem, os professores, diretores e responsáveis pelos alunos podem tomar ações em conjunto para alcançar uma melhora pontual e global dos alunos analisados.

\section{Descrição do jogo: SpaceGEMS}

Nesta seção são apresentadas a temática, mecânicas e arquitetura por trás do jogo SpaceGEMS, proposto como principal meio de captura de áudios.

\subsection{Tema e Mecânicas}

O SpaceGEMS se passa em um futuro muito distante, na galáxia TLK 2M3, na qual os alunos são pilotos contratados por grandes megacorporações de entregas espaciais. Eles 
são responsáveis por colher mercadorias em um planeta e entregar em outro, recebendo créditos (pontos) em troca. A empresa (time de alunos) que acumular mais créditos ao longo de um número pré-determinado de turnos, é a vencedora da partida.

A representação da galáxia é feita inicialmente de forma que a maioria dos planetas possuam quatro vizinhos. Entretanto alguns podem estar nos limites e possuir obstáculos circundantes, diminuindo esse número a pelo menos duas opções. Cada jogador é representado por um foguete, com a cor do seu time e seu nome, além de uma indicação da sua carga atual para cada bem. Na sua vez, o jogador deve realizar três movimentos com seu foguete, movendo-se para planetas vizinhos ao atual.

A dinâmica do jogo é baseada no mecanismo de pegar e entregar (do inglês, Pick up and Delivery). O espaço de jogo é divido em setores com planetas que podem produzir bens, representados por três pedras preciosas: rubis, esmeraldas e safiras ou demandas de clientes, representadas por uma placa com o desenho do bem e um preço pago. Se o jogador chega em um planeta com um conjunto de bens disponíveis, ele colhe esses bens para seu foguete. Se o jogador chega em um planeta com um conjunto de demandas, ele descarrega os respectivos bens e recebe créditos (pontos).

A divisão em grupos automaticamente estimula a comunicação entre os membros para traçar melhores abordagens para a solução do problema e evitar a inibição do indivíduo. O jogo tem o perfil de interação indireta entre jogadores: não há ataques, troca involuntária ou revés no estado do jogo. Não há eliminação de jogadores e parte da pontuação é computada apenas ao final da partida, na busca que todos continuem engajados até a conclusão e criando um clímax para o resultado final.

\subsection{Dinâmica de aplicação}

A partida é planejada previamente pelo professor, através do site da aplicação, cadastrando os nomes dos alunos e selecionando o conjunto de palavras a ser utilizado. $\mathrm{O}$ sistema separa os alunos em grupos e os espalha pela galáxia aleatoriamente, igualmente para a disponibilidade de bens e demandas. O professor também define uma duração da partida, que é função do número de turnos que cada aluno irá participar.

No dia da atividade, o professor utiliza um smartphone como controle do jogo e, adicionalmente, uma TV ou tela geral apresenta o estado geral do jogo para todos os participantes (os alunos podem visualizar esse estado também através de seus telefones). Após instruções iniciais dadas pela professora, o jogo entra em um laço até que todos os alunos tenham pronunciado as palavras presentes na lista pré-selecionada. A cada iteração, alternando entre os grupos criados, uma criança é selecionada e deve realizar três movimentos, escolhendo e pronunciando a palavra associada para cada movimentação possível para seu foguete dentro do jogo.

Quando o número de rodadas determinado é concluído, o jogo finaliza anunciando qual grupo é o vencedor. Posteriormente, o professor tem acesso, via site, ao desempenho dos alunos na pronúncia de cada palavra, bem como palavras que foram mais erradas pelos alunos e fonemas que foram frequentemente pronunciados incorretamente. Assim, pode tomar ações macro e micro com relação às dificuldades encontradas. 
VIII Congresso Brasileiro de Informática na Educação (CBIE 2019)

Anais do XXX Simpósio Brasileiro de Informática na Educação (SBIE 2019)

\subsection{Arquitetura}

Uma visão geral da arquitetura do software proposto pode ser visto na Figura 1. Os componentes destacados são Módulo de ASR, Módulo de Configuração, Módulo de Controle (smartphone), Cliente Público, Módulo de Resultados e Servidor de Jogo.

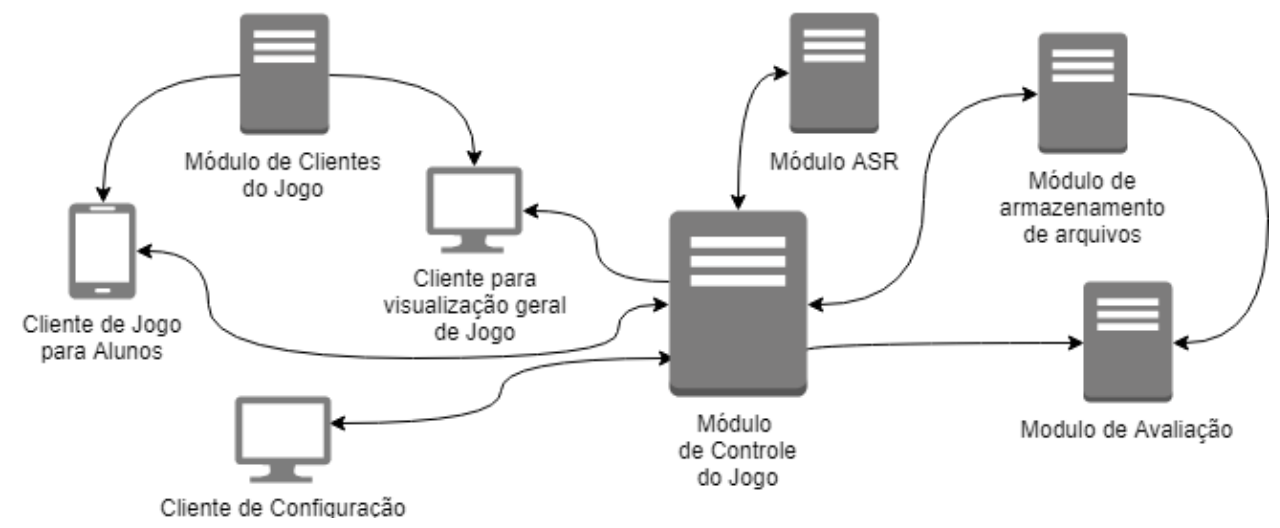

Figura 1. Visão geral da arquitetura do SpaceGEMS

O Módulo ASR é responsável por receber uma palavra por escrito e arquivo de áudio com a leitura realizada pelo aluno. O serviço realiza a transcrição do áudio e confere se a mesma é igual a palavra que foi pronunciada. O serviço ASR é especializado na avaliação da leitura e permite levantar informações sobre os fonemas pronunciados pela criança e os fonemas que deveriam ser pronunciados, de forma que o professor possa ter informações ricas, por exemplo, sobre quais fonemas têm sido pronunciados incorretamente ou quais tem sido frequentemente trocados por outros.

O Módulo de Controle de Jogo é responsável por gerir completamente o andamento das partidas, registrando os jogadores, os seus elementos de jogabilidade e as ações solicitadas pelos clientes de jogo. Também é responsável por toda a orquestração dos demais serviços e integração direta com os clientes. A cada ação de um jogador recebida, ele verifica se é uma ação válida, recebe o áudio capturado no cliente, envia o arquivo para o Serviço de armazenamento de arquivos, gera um acesso ao Serviço de ASR para avaliar a resposta do jogador e avança o estado do jogo.

O Cliente de Configuração é fornecido pelo Servidor de Controle do Jogo para que o professor responsável pela atividade possa cadastrar os nomes dos alunos, configurar a duração da partida e selecionar o conjunto de palavras que serão lidas como parte das ações dos jogadores. Nesse sentido, o professor tem liberdade para selecionar palavras sobre temas específicos ou palavras que os alunos já mostraram certa dificuldade ao longo da disciplina, tornando a abordagem proposta adaptável a diferentes cenários. Atualmente ele é desenvolvido como um conjunto de páginas dinâmicas, acessadas diretamente no servidor.

O Módulo de Clientes de Jogo é responsável por fornecer os meios de acesso necessários dentro da atividade. Estão disponíveis dois clientes: um cliente SPA (Single Page Application que pode ser acessado por qualquer navegador em um dispositivo móvel ou estação de trabalho e um cliente Android. Ambos os clientes possuem as mesmas funcionalidades mas o cliente em Android tem o objetivo de ser visualmente mais atraente e com mais recursos de animação. Cada cliente pode ser utilizado no modo ativo como 
VIII Congresso Brasileiro de Informática na Educação (CBIE 2019)

Anais do XXX Simpósio Brasileiro de Informática na Educação (SBIE 2019)

Cliente de Jogo (permite enviar comandos ao Servidor de Jogo) ou como apenas no modo passivo como Cliente de visualização do estado atual.

O Módulo de armazenamento de arquivos é responsável por receber os arquivos de áudios e metadados coletados durante a realização de uma partida. Sua responsabilidade é servir como memória a longo prazo de todos os dados colhidos de forma que novas aplicações de análise possam ser construídas para operar os dados colhidos previamente.

O Módulo de Avaliação é responsável por avaliar as jogadas, os resultados do ASR, os arquivos de áudio e as informações colhidas durante e após uma partida. Sua responsabilidade é gerar relatórios para o professor responsável que dizem respeito ao desempenho de cada aluno e ao desempenho geral da turma. Isso permite que o professor consiga ter mais informações sobre quais fonemas, palavras ou questões de pronúncia e leitura que devem ser abordados nas aulas futuras.

\section{Prova de Conceito}

Nesta seção são apresentados os experimentos realizados para verificar a qualidade da abordagem. Foi realizada uma simulação controlada de utilização da abordagem com o intuito de avaliar seu comportamento e sua capacidade de reproduzir resultados confiáveis. As análises macro dos resultados em forma de rankings de pontuação dos alunos e palavras mais pronunciadas correta e incorretamente são analisados. A abordagem foi testada com um conjunto de 879 áudios reais de crianças em fase de alfabetização fornecido pelo CAEd/UFJF (Centro de Políticas Públicas e Avaliação da Educação ${ }^{1}$ ) . Cada áudio contém a leitura de até 50 palavras, as quais podem ter sido pronunciadas correta ou incorretamente, e contém leitura de uma criança diferente, não havendo repetição de locutores. No total são consideradas 35934 pronúncias de palavras.

Os áudios foram submetidos palavra por palavra ao SpaceGEMS como se fossem alunos respondendo em tempo real na sala de aula e a correção realizada pelo módulo de ASR foi comparada com a correção efetuada pelos avaliadores do CAEd/UFJF. O sistema de avaliação automático acerta exatamente a leitura das crianças em $86 \%$ das palavras (acurácia). Destaca-se a precisão de $97 \%$ e cobertura de $88 \%$ para detecção de leitura certa, de forma que o SpaceGEMS represente um classificador de leitura que são de fato corretas e encontra a maior parte das pronúncias corretas.

A pontuação alcançada por cada jogador foi calculada de acordo com seus erros e acertos e comparadas com a pontuação que o jogador alcançaria na abordagem automática e sua nota real. Nas duas pontuações, cada erro conta como um ponto e não há penalidade nos erros para motivar o aluno. A Figura 2 apresenta os erros de pontuação. Percebese que a maioria dos áudios estão concentrados ao redor do erro zero, ou seja, possuem pontuação automática exatamente igual à manual. Nos casos onde a pontuação se afasta do zero, é possível notar que há uma tendência do SpaceGEMS não pontuar de forma excessiva, ou seja, geralmente gerando pontuação menor que o esperado, contribuindo para que erros de leitura tenham menos possibilidade de serem considerados acertos.

O professor pode adquirir uma visão global da turma analisando a média, mediana e moda das pontuações obtidas pelos alunos da turma. Nesse sentido, as métricas são apresentadas na Tabela 1 , tanto para o resultado manual quanto para o resultado au-

\footnotetext{
${ }^{1}$ http://www.caed.ufje.br/
} 
VIII Congresso Brasileiro de Informática na Educação (CBIE 2019)

Anais do XXX Simpósio Brasileiro de Informática na Educação (SBIE 2019)

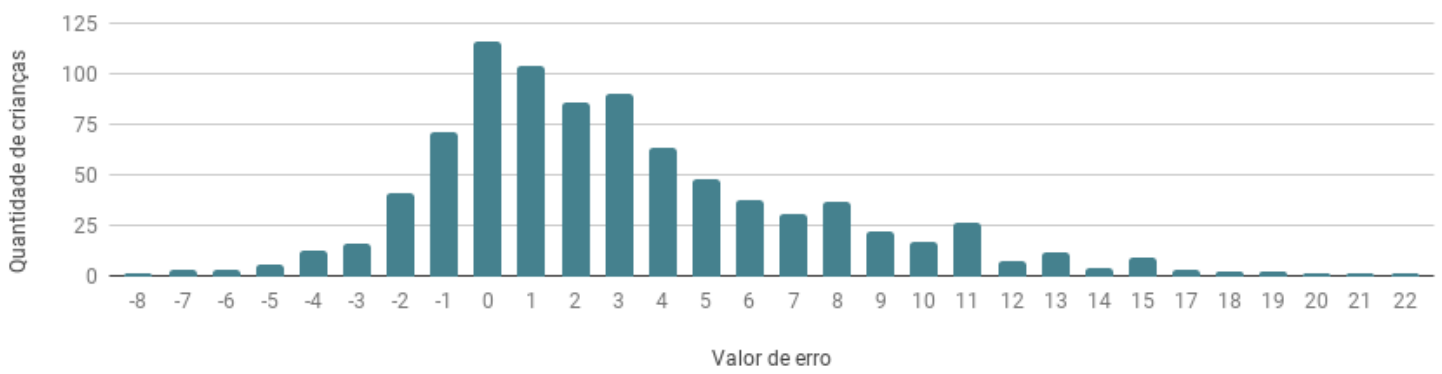

Figura 2. Distribuição do erro de pontuação atribuídos dentro da prova de conceito com áudios previamente capturados.

tomático. Percebe-se que a abordagem é capaz de obter resultados gerais muito próximos do resultado manual, dando indícios para o professor de quão variado é sua turma e de qual é o desempenho médio dos alunos.

Tabela 1. Métricas de avaliação do desempenho geral da turma

\begin{tabular}{|c|c|c|}
\hline Métricas & Manual & Abordagem \\
\hline Média & 39,15 & 36,01 \\
\hline Desvio padrão & 11,52 & 11,99 \\
\hline Mediana & 43 & 40 \\
\hline Moda & 45 & 45 \\
\hline
\end{tabular}

Além da pontuação, é possível comparar o ranking de palavras mais comumente lidas incorretamente e mais comumente lidas corretamente. A interseção entre as palavras mais comumente lidas corretamente e as palavras mais comumente lidas incorretamente para 10, 20 e 30 palavras pode ser visualizada na Tabela 2. Existe uma boa interseção para as palavras mais acertadas, mas a interseção entre as palavras mais erradas precisa ser melhorada. Nesse sentido, questões técnicas como ambiente de gravação e qualidade do equipamento, que não foram controladas pelos autores, influenciam negativamente na identificação de alguns áudios.

Tabela 2. Interseção entre palavras mais acertadas e mais erradas segundo avaliação manual e automática.

\begin{tabular}{|l|l|l|}
\hline Número de palavras & Interseção entre mais acertadas & Interseção entre mais erradas
\end{tabular}

\begin{tabular}{|c|c|c|}
\hline 10 & $60 \%$ & $20 \%$ \\
\hline 20 & $60 \%$ & $25 \%$ \\
\hline 30 & $74 \%$ & $27 \%$ \\
\hline
\end{tabular}

\section{Conclusão e trabalhos futuros}

Este trabalho teve por objetivo propor uma abordagem computacional gamificada para detecção de falhas na alfabetização através de técnicas de reconhecimento automático de fala. Os trabalhos na literatura apontam extenso uso dessas técnicas no aprendizado de uma língua estrangeira, mas pouco esforço é empregado para o desenvolvimento da língua 
VIII Congresso Brasileiro de Informática na Educação (CBIE 2019)

Anais do XXX Simpósio Brasileiro de Informática na Educação (SBIE 2019)

materna. Nesse sentido, propõe-se a abordagem Talk2Me e implementa-se o jogo SpaceGEMS para auxiliar professores e gestores na identificação de problemas de fluência, possibilitando a tomada de decisões.

Após a prova de conceito da abordagem com um conjunto de áudios captados de maneira controlada, fora de uma atividade em sala de aula, um trabalho futuro é a aplicação e avaliação da abordagem em sala de aula. Dessa forma, os professores podem validar os resultados encontrados e avaliar as informações levantadas pelo sistema, pontuando o que é mais pertinente para que decisões de planejamento possam ser tomadas. A aplicação em sala de aula permite identificar a necessidade de aprimoramento do ASR, adição de novos artifícios de gamificação e nível de interesse dos alunos no jogo.

Como trabalhos futuros, escolas da cidade de CAEd/UFJF serão convidadas para experimentar o jogo em suas aulas. Será realizado um experimento conduzido pelos autores para verificar a interação dos alunos e professores na condução da avaliação. Os dados serão disponibilizados para a direção da escola. Por fim, a arquitetura da solução poderá ser estendida em duas frentes: (1) para tratar outros problemas identificáveis no início da alfabetização, como problemas no trato vocal e dislexia, (2) para tratar outros aspectos da oralidade, como a capacidade de leitura e compreensão de textos.

\section{Agradecimentos}

Agradecemos ao CAEd/UFJF pelo financiamento do projeto e disponibilização da base de avaliação utilizada nos experimentos.

\section{Referências}

Anwar, A., Rahman, M. M., Ferdous, S., Anik, S. A., and Ahmed, S. I. (2011). A computer game based approach for increasing fluency in the speech of the autistic children. In Advanced Learning Technologies (ICALT), 2011 11th IEEE international conference on, pages 17-18. IEEE.

Barrére, E., Vitor, M. A., and de Almeida, M. A. (2017). Ampliação das possibilidades de gamificação no moodle. In Brazilian Symposium on Computers in Education (Simpósio Brasileiro de Informática na Educação-SBIE), volume 28, page 605.

Celestino, P. G. (2019). A oralidade infantil e desenvolvimento cognitivo à partir da prática docente. Revista Internacional de apoyo a la inclusión, logopedia, sociedad y multiculturalidad, 5(1).

Dallabona, S. R. and Mendes, S. M. S. (2004). O lúdico na educação infantil: jogar, brincar, uma forma de educar. Revista de divulgação técnico-científica do ICPG, 1(4):107112.

Deterding, S., Dixon, D., Khaled, R., and Nacke, L. (2011). From game design elements to gamefulness: defining gamification. In Proceedings of the 15th international academic MindTrek conference: Envisioning future media environments, pages 9-15. ACM.

Dias, N. M., Leão, C. B. R., Pazeto, T. d. C. B., Martins, G. L. L., Pereira, A. P. P., and Seabra, A. G. (2016). Avaliação da leitura no Brasil: revisão da literatura no recorte 2009-2013. Psicologia: teoria e prática, 18:113 - 128. 
VIII Congresso Brasileiro de Informática na Educação (CBIE 2019)

Anais do XXX Simpósio Brasileiro de Informática na Educação (SBIE 2019)

Fuchs, L. S., Fuchs, D., Hosp, M. K., and Jenkins, J. R. (2001). Oral reading fluency as an indicator of reading competence: A theoretical, empirical, and historical analysis. Scientific studies of reading, 5(3):239-256.

Hudson, R. F., Lane, H. B., and Pullen, P. C. (2005). Reading fluency assessment and instruction: What, why, and how? The Reading Teacher, 58(8):702-714.

Liu, Q., Cai, F., Yang, Y., and Han, T. (2015). Gamification design based research on speech training system for hearing-impaired children. In International Conference on Engineering Psychology and Cognitive Ergonomics, pages 140-151. Springer.

MEC (2018). Base nacional comum curricular. http://basenacional comum. mec.gov.br/wp-content/uploads/2018/02/bncc-20dez-site. pdf. Acessado: 30-01-2019.

Musti-Rao, S., Lo, Y.-y., and Plati, E. (2015). Using an ipad® app to improve sight word reading fluency for at-risk first graders. Remedial and Special Education, 36(3):154166.

Rocha, P. S. V. d. S. (2018). A importância do lúdico na educação infantil: uma análise a partir da concepção de professores.

Santos, J. d. A. d. and Freitas, A. L. C. d. (2017). Gamificação aplicada a educação: Um mapeamento sistemático da literatura. Renote-Revista Novas Tecnologias na Educação, Porto Alegre, 15:0-0.

Saputra, M. R. U. (2015). Lexipal: Design, implementation and evaluation of gamification on learning application for dyslexia. Int. J. Comput. Appl, 131:37-43.

Soares, E., Carlos Carchedi, L., Gomes Jr, J., Barrere, E., and Souza, J. (2018). Avaliação automática da fluência em leitura para crianças em fase de alfabetização. page 11.

Suehiro, A., de Souza Benfica, T., and Cardim, N. (2009). Produção científica sobre o teste desenho da figura humana entre 2002 e 2012. Psicologia: Ciência e Profissão, 36:439-448.

Takita, T., Akimoto, K., and Hoshino, J. (2017). Reading aloud training game for children with auto evaluation of oral reading fluency. In International Conference on Entertainment Computing, pages 404-408. Springer.

Tejedor-García, C., Escudero-Mancebo, D., González-Ferreras, C., Cámara-Arenas, E., and Cardeñoso-Payo, V. (2016). Tiptoptalk! mobile application for speech training using minimal pairs and gamification. 